\title{
Demonstration of Chlamydia trachomatis in colposcopic cervical biopsy specimens by an immunoperoxidase method
}

\author{
J M Edwards, A R Campbell, A Tait, M Lusher
}

\begin{abstract}
A total of 31 cervical biopsy specimens were taken from 29 women attending a genitourinary medicine clinic, nine women (11 biopsy specimens) were known to have Chlamydia trachomatis cervicitis and 20 women were known to be free of chlamydial infection. The specimens were routinely processed to paraffin wax and stained by an antiChlamydia immunoperoxidase technique to localise the organisms. Of the 11 positive biopsy specimens three showed positive staining of elementary/reticulate bodies. In one case the surface endocervical cells showed large inclusions which were packed with chlamydial bodies.

The diagnosis of chlamydial infection is difficult to make clinically and in routine cytological and histological specimens but immunoperoxidase staining can clearly identify $C$ trachomatis inclusions in cervical biopsy specimens provided infection is severe.
\end{abstract}

Infections caused by Chlamydia trachomatis are now recognised as the most prevalent and among the most damaging of all sexually transmitted diseases. ${ }^{1}$ Infection is often low grade and chronic but is an important cause of pelvic inflammatory disease and its sequelae. Treatment is simple and effective but signs and symptoms are often subtle.

Papanicolou stained cervical smear cell changes are now regarded as too non-specific to allow Chlamydia to be differentiated from other cervical infections. ${ }^{2}$ Histological features associated with chlamydial infection in cervical biopsy specimens have recently been described $^{3}$ and their specificity awaits further confirmation. Use of electron microscopy to search for inclusions has a detection rate of only $4 \%{ }^{4}$

Fluorescent antibody staining of cervical smears is nearly as sensitive as culture provided the smear is taken from the endocervix ${ }^{5}$ but fluorescent antibody staining of cervical biopsy specimens is less sensitive. ${ }^{3}$ A peroxidase-antiperoxidase method has been used on cervical smears with some success in localising inclusions. ${ }^{6} \mathrm{We}$ attempted to stain for $C$ trachomatis inclusions in paraffin wax embedded cervical biopsy specimens using an immunoperoxidase adaptation of the fluorescence technique to give a more convenient and more permanent result.

\section{Methods}

Endocervical swabs were taken from nulliparous, adult women attending a genitourinary medicine clinic. The swabs were immediately transferred to the laboratory in Chlamydia culture medium and an enzyme linked immunosorbent assay (ELISA) test was performed to detect the group specific lipopolysaccharide antigen (LPS) (Antigenz, Northumbria Biologicals Ltd, Newcastle). All positive ELISA cases were confirmed with direct fluorescence (Microtrak, Syva). Eleven known positive and 20 known negative cases were brought back to the clinic one week later and a colposcopically directed cervical biopsy specimen was taken from the squamocolumnar junction.

The specimens were fixed in formalin and processed to paraffin wax as normal. A routine haematoxylin and eosin section was examined to check that all biopsy specimens included the squamocolumnar junction.

Two monoclonal antibodies were used on the 31 cervical biopsy specimens plus several infected and non-infected McCoy cell monolayer cultures. One method used the genus specific monoclonal antibody LPS (NovoBiolabs) which is the same antigen as is detected in the ELISA technique performed in our laboratory. The other used the species specific monoclonal antibody MOMP (Syva Company, USA) which recognises the major outer membrane protein of $C$ trachomatis elementary bodies. ${ }^{7}$ Both the commercially available LPS and MOMP monoclonal mouse antibodies are labelled with fluorocien isothiocyanate (FITC). We used two separate immunocytochemistry systems to convert to an immunoperoxidase end point, a 1 in 100 rabbit anti-mouse secondary antibody and a 1 in 100 mouse anti-FITC monoclonal secondary. An avidin-biotin-complex (ABC) system was then used to bypass the FITC signal and visualised using peroxidase and diaminobenzidine (DAB). Both these systems gave identical results.

All immunocytochemical experiments included positive and negative control slides. To begin with the positive control consisted of infected McCoy cell monolayers. After positive results were achieved on paraffin wax sections these cases were used as positive controls. The negative control was performed by omitting the primary antibody from the protocol.

\section{Results}

Both monoclonal antibodies LPS and MOMP 


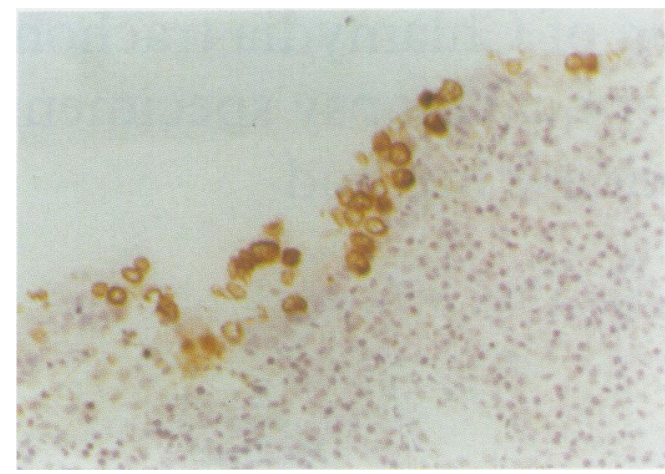

Figure 1 Cervical biopsy specimen stained by an immunoperoxidase stain to illustrate chlamydial bodies on the surface and within endocervical cells.

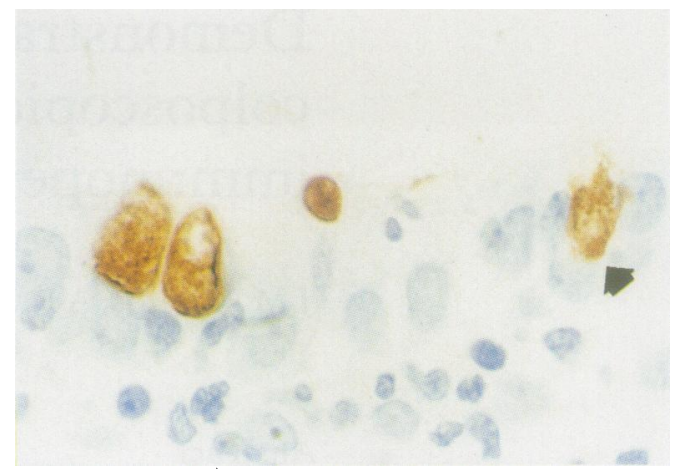

Figure 2 Chlamydial inclusions are stained brown within endocervical cells and one cell (arrow) is in the process of releasing its chlamydial bodies onto the surface. showed infected cells in monolayer cultures. The LPS antibody failed to identify any evidence of infection in the 11 positive biopsy specimens. The MOMP technique detected three positive specimens: in two, chlamydial bodies were present free on the endocervical cell surface; in the third case (fig 1) a large number of chlamydial inclusions were identified on the surface and within the endocervical cells lining the canal (fig 2). In one cell (arrowed) the inclusion appeared to be about to release its bodies on to the surface. The poor sensitivity was disappointing, so we compared the positivity of the ELISA technique performed at the initial visit with our results and we noted that our stains were only picking up the patients who were reported as having a strongly positive ELISA result. The case was scored as ++++ , two cases as +++ ; the six remaining Chlamydia cases had less strong ELISA results, with three cases ++ and three as + .

\section{Discussion}

We were unable to find a readily available immunoperoxidase labelled antibody and therefore attempted to localise $C$ trachomatis by an adaptation of an immunofluoresence technique, as described. We confirm the generally accepted view that the endocervical cells provide a desirable environment for the organism to multipy whereas the squamous cells and connective tissue cells were spared. We found only the columnar cells on the endocervical surface were infected whereas the columnar cells within the crypts did not contain inclusions, and this finding differs from that of other workers. ${ }^{3}$

The LPS antibody stained the infected cells in the monolayers but failed to stain the positive biopsy specimens. The cells were fixed for about 10 minutes whereas fixation time for the biopsy specimens varied from one to six weeks. We reduced the fixation time to 24 hours but the positive rate failed to improve. Lundermose et $a^{8}$ stained $C$ psittaci using an LPS antibody on formalin fixed necropsy lung tissue. In the light of their study our failure with anti-LPS was dis- appointing (table). The MOMP antibody was also positive on all infected monolayer cultures, our pick-up rate on biopsy specimens of three out of 11 corresponds closely to earlier work using a fluorescein labelled MOMP antibody in which two cases out of 28 showed chlamydial inclusions and one showed elementary bodies attached to the surface of the glandular epithelium. ${ }^{3}$ Direct fluorescence antibody testing on smears has shown that the species specific MOMP antibody produces more intense fluorescence than the genus specific anti-LPS monoclonal antibody ${ }^{9}$ and we have also shown that MOMP is superior.

$C$ trachomatis activity waxes and wanes so perhaps in some of our positive cases very few organisms were present at the time of biopsy. We attempted to minimise this problem by performing the index endocervical swab to identify the infected group and the biopsy a week apart; a shorter period was logistically impossible. We acknowledge the fact that our case and control numbers are small and that this curtails serious assessment of sensitivity and specificity, but the close correlation with the degree of positivity of the ELISA technique suggests that our immunoperoxidase stain gives good identification of chlamydial bodies provided infection is severe. The advantages are that tissue morphology is preserved, interpretation of the exact infection site is simple, and the stain is less transient and more convenient to assess than a fluorescence technique.

Our thanks to Dr S J Richmond, Department of Virology, University of Manchester, for her help and encouragement, and also to Dr B Pratt, Medical Microbiology Department, and also to Dr B Pratt, Medical Microbiology Department, University of Liverpool, and Dr I iAcDicken,
Histopathology, Women's Hospital, Liverpool.

Correlation between ELISA result and cervical biopsy immunoperoxidase staining in 29 patients

\begin{tabular}{lll}
\hline Case No & ELISA (LPS) & $\begin{array}{l}\text { Immunoperoxidase } \\
\text { (MOMP) }\end{array}$ \\
\hline 1 & ++++ & Inclusions \\
2 & +++ & Free bodies \\
3 & +++ & Free bodies \\
$4,5,6$ & ++ & Negative \\
$7,8,9$ & + & Negative \\
$10-29$ & Negative & Negative \\
\hline
\end{tabular}


1 US Department of Health and Human Services. Chlamydia trachomatis infections. Policy guidelines for prevention and control. US Department of Health and Human Services. Atlanta, Georgia: Centers for Disease Control, 1985.

2 Bernal JN, Martineq MA, Dabancens A. Evaluation of proposed cytomorphologic criteria for the diagnosis of Chlamydia trachomatis in Papanicolaou smears. Acta Cytol 1988;33:309-13.

3 Kiviat NB, Paavonen JA, Wolner-Hansen P, et al. Histopathology of endocervical infection caused by Chlamydia trachomatis, herpes simplex virus, Trichomonas vaginali and Neisseria gonorrhoeae. Hum Pathol 1990;21:831-7.

4 Dunlop EMC, Garner A, Darougar S, Treharne JD, Woodland RM. Colposcopy, biopsy, and cytology results in women with chlamydial cervicitis. Genitourin Med
1989;65:22-31.

5 Kiviat NB, Peterson M, Kinney-Thomas E, Tam M, Stamm WE, Holmes KK. Cytological manifestations of cervical and vaginal infections. $J A M A$ 1985;253:997-1000

6 Leopardi $O$, Celentano C, Naughten $W$. Immunoperoxidase demonstration of chlamydia inclusions in routine Pap

7 Caul EO, Paul ID, Milner JD, Crowley T. Non-invasive sampling method for detecting Chlamydia trachomatis. Lancet 1988;ii:1246-7.

8 Lundermose AG, Lundemose JB, Birkelund S, Christiansen $G$. Detection of chlamydia in postmortal formalin fixed tissue. APMIS 1989;97:68-74.

9 Barnes RC. Laboratory diagnosis of human chlamydial infections. Clin Microbiol Rev 1989;2:119-36.

\title{
Evaluation of a screening test for detecting urinary tract infection in newborns and infants
}

\author{
B Lejeune, R Baron, B Guillois, D Mayeux
}

Microbiology and
Public Health
Laboratory, CHU
Morvan - 29609 Brest
Cedex France
B Lejeune
R Baron
Department of
Paediatrics, CHU
Morvan
B Guillois
Medical Computer
Laboratory, Faculty of
Medecine, Les Nancy
France
D Mayeux
Correspondence to:
Dr B Lejeune
Accepted for publication 22
May 1991

\begin{abstract}
The results of a study of a screening test for urinary tract infection (UTI) in infants under 18 months is reported. Two hundred and forty three urine specimens were tested in the laboratory using AMES Multistix 8SG reagent strips read by photometer. The strips included three potential markers for urinary tract infection: leucocyte esterase, nitrite, and protein. The predictive value of a positive result (PPV) was low. The predictive value of negative test (NPV) when combining the screen of leucocyte esterase, nitrite, and protein was $99.4 \%$ with no difference between boys and girls. The test for leucocyte esterase had a $97.6 \%$ negative predictive value. An examination of the results by age confirms the good NPV in all age groups.

Paediatricians should find Multistix 8SG strips a useful aid in the diagnosis of urinary tract infection in infants, and that costly culture of samples with negative strip tests can be avoided.
\end{abstract}

Boreland and colleagues reported the diagnostic value of a screening test for urinary tract infection in paediatric inpatients using three tests: nitrite, blood, and protein. ${ }^{1}$ We studied consecutive urine samples from 243 neonates and infants under 18 months: 85 were less than 28 days old; 81 aged between 21 and 182 days; and 77 were more than 182 days old. Our aim was to identify the dipstick test which gave the highest diagnostic accuracy in routine practice for this age group and to evaluate the leucocyte esterase test.

\section{Method}

Urine reagent strips for nitrite, leucocyte esterase, and protein were used (Multistick 8 SG AMES) and read by the Clinitek System photometer (AMES). All screening tests were performed by the same investigator in our laboratory. All samples were microscopically examined, cultured, and bacteria enumerated (DGU Institut Pasteur Production, France). The criteria for diagnosis of urinary tract infection ${ }^{2}$ were as follows: a combination of a white cell count of $>25 \times 10^{9} / 1$ for boys or 50 $\times 10^{9} / 1$ for girls under 8 days of age; $>10 \times 10^{9} / 1$ for those older than 8 days; and a bacterial count of $>10^{5} \mathrm{ml}^{-1}$ with a maximum of two bacterial species. Samples with $10^{4} / \mathrm{ml}^{-1}$ or less, or those with more than two bacterial species were considered contaminated.

The statistical analysis of the screening tests results was based on Baye's tests and compared with the criteria for diagnosis of urinary tract infection.

\section{Results}

Thirty seven $(15.2 \%)$ specimens met the criteria for bacteriological infection, and 81 $(33 \%)$ were regarded as contaminated. One hundred and forty six $(60 \%)$ specimens were negative by the strip test and 97 were positive for one or more of leucocyte esterase, nitrate, or protein. The percentage of false negative results was $1.6 \%$ (four urine specimens) and was attributed to asymptomatic infection $\left(>10^{5} / \mathrm{ml}^{-1}\right)$ after review of the medical records.

The negative predictive value (NPV) obtained with the combination of leucocyte esterase and protein, and with the combination of leucocyte esterase, nitrite, and protein 